\title{
Distinct functions of transforming growth factor- $\beta$ signaling in c-MYC driven hepatocellular carcinoma initiation and progression
}

Haichuan Wang $\mathbb{1}^{1,2,3}$, Pan Wang ${ }^{2}$, Meng Xu ${ }^{4}$, Xinhua Song ${ }^{2}$, Hong Wu${ }^{1}$, Matthias Evert ${ }^{5}$, Diego F. Calvisi ${ }^{5}$, Yong Zeng $\mathbb{B}^{1,3}$ and Xin Chen $\mathbb{D}^{2}$

\begin{abstract}
Dysregulation of transforming growth factor-beta (TGF $\beta$ ) signaling has been implicated in liver carcinogenesis with both tumor promoting and inhibiting activities. Activation of the c-MYC protooncogene is another critical genetic event in hepatocellular carcinoma (HCC). However, the precise functional crosstalk between c-MYC and TGF $\beta$ signaling pathways remains unclear. In the present investigation, we investigated the expression of TGF $\beta$ signaling in c-MYC amplified human HCC samples as well as the mechanisms whereby TGF $\beta$ modulates c-Myc driven hepatocarcinogenesis during initiation and progression. We found that several TGF $\beta$ target genes are overexpressed in human HCCs with c-MYC amplification. In vivo, activation of TGF $\beta 1$ impaired c-Myc murine HCC initiation, whereas inhibition of TGF $\beta$ pathway accelerated this process. In contrast, overexpression of TGF $\beta 1$ enhanced c-Myc HCC progression by promoting tumor cell metastasis. Mechanistically, activation of TGF $\beta$ promoted tumor microenvironment reprogramming rather than inducing epithelial-to-mesenchymal transition during HCC progression. Moreover, we identified PMEPA1 as a potential TGF $\beta 1$ target. Altogether, our data underline the divergent roles of TGF $\beta$ signaling during C-MYC induced HCC initiation and progression.
\end{abstract}

\section{Introduction}

Hepatocellular carcinoma (HCC) is the most common primary liver malignancy and a leading cause of cancerrelated deaths worldwide ${ }^{1}$. Most HCC patients are diagnosed at an advanced stage and current treatments show unsatisfactory therapeutic efficacy ${ }^{2}$. Generally, HCC develops in the context of a diseased liver through a multistep process. Etiologic factors including chronic hepatitis $B$ and $C$ virus infection, aflatoxin exposure, and heavy alcohol consumption contribute to cycles of hepatocyte damage/cell death and compensatory regeneration ${ }^{3}$.

\footnotetext{
Correspondence: Yong Zeng (zengyong@medmail.com.cn) or Xin Chen (xin. chen@ucsf.edu)

'Liver Transplantation Division, Department of Liver Surgery, West China Hospital, Sichuan University, Chengdu, People's Republic of China ${ }^{2}$ Department of Bioengineering and Therapeutic Sciences and Liver Center, University of California, San Francisco, CA, USA

Full list of author information is available at the end of the article Edited by M. Agostini
}

These events, together with the progressive accumulation of genetic and epigenetic changes induces a "field defect" in the liver parenchyma, prone to malignant conversion and tumor initiation ${ }^{4}$. The pathophysiology of HCC might be thoroughly divergent during the initiation and progression stages ${ }^{5}$. Thus, there is a need to better delineate the distinct molecular pathways regulating HCC initiation and progression to develop innovative and effective diagnostic and therapeutic approaches.

Dysregulation of the transforming growth factor-beta (TGF $\beta$ ) signaling is a critical tumorigenic event contributing to hepatocarcinogenesis ${ }^{6}$. TGF $\beta 1$, one of the three different homodimer TGF $\beta$ isoforms (TGF $\beta 1$, TGF $\beta 2$ and TGF $\beta 3$ ), is a key member of the TGF $\beta$ superfamily ${ }^{7}$. In most cell types, the TGF $\beta 1$ ligand binds to type I/II TGF $\beta$ receptor (T $\beta R I / I I)$ to induce phosphorylation of mothers against decapentaplegic homolog 2 and 3 (Smad2 and Smad3; activin/TGF $\beta$ specific R-Smads) ${ }^{8}$. Activated R-Smads form

\section{(c) The Author(s) 2021}

(c) (i) Open Access This article is licensed under a Creative Commons Attribution 4.0 International License, which permits use, sharing, adaptation, distribution and reproduction c. in any medium or format, as long as you give appropriate credit to the original author(s) and the source, provide a link to the Creative Commons license, and indicate if changes were made. The images or other third party material in this article are included in the article's Creative Commons license, unless indicated otherwise in a credit line to the material. If material is not included in the article's Creative Commons license and your intended use is not permitted by statutory regulation or exceeds the permitted use, you will need to obtain permission directly from the copyright holder. To view a copy of this license, visit http://creativecommons.org/licenses/by/4.0/. 
hetero-oligomeric complexes with a common-partner (co-) Smad, denominated Smad4. The TGF 1 1-Smad signaling has been demonstrated to play a critical role in the development of liver cancer ${ }^{9}$. In HCC, TGF $\beta 1$ is a bifunctional regulator that either inhibits or promotes hepatocarcinogenesis mainly depending on the tumor stage ${ }^{10,11}$. In particular, TGF $\beta 1$ has been shown to be involved in regulating tumor proliferation, epithelial-to-mesenchymal transition (EMT), and tumor microenvironment during HCC progression and metastasis ${ }^{12}$.

Activation of c-MYC is an important oncogenic event during hepatocarcinogenesis. In humans, amplification of c-MYC has been found in $27 \%$ HCC patients ${ }^{13}$. The oncogenic potential of c-Myc has been demonstrated by the finding that its overexpression in the mouse liver is sufficient to trigger HCC formation ${ }^{14,15}$. c-MYC has been also shown to directly interact with SMAD2/3 to inhibit TGF- $\beta$ signaling ${ }^{16}$, thus promoting cell growth and cancer development. However, the crosstalk between c-MYC and TGF $\beta$-SMADs signaling pathways during HCC initiation and progression remains poorly defined.

Here, we investigated the activation of TGF $\beta$ signaling in c-MYC amplified human HCC samples. In addition, we determined how the TGF $\beta$ cascade modulates c-Myc driven HCC initiation and progression. Our results indicate that TGF $\beta$ may function to inhibit $\mathrm{HCC}$ initiation but promote metastasis during $\mathrm{HCC}$ progression.

\section{Materials and methods}

\section{Constructs and reagents}

The constructs used for mouse injection in this study, including pT3 (Vector), pT3-EF1a-TGF $\beta 1$ (mouse), pT3EF1a-c-Myc (mouse), pCMV, pCMV-Cre, and pCMVSleeping Beauty transposase (SB), were previously descri$\operatorname{bed}^{14}$. The antisense oligos targeting mouse Smad2 and Smad3 were kind gifts from Dr. Simon W. Ro (Kyung Hee University, Seoul, Republic of Korea) ${ }^{17}$. The antisense oligos targeting mouse Smad4 were designed and generated as previously described ${ }^{18}$. Sequence information for the antisense oligos are: shSmad2, 5'- TGCTGTTGACAGTG AGCGACCGCAGTTAGCTGTGGACTTATAGTGAAGC C-ACAGATGTATAAGTCCACAGCTAACTGCGGGTGC CTACTGCCTCGGA -3'; shSmad3, 5'- TGCTGTTGAC AGTGAGCGATAGCTTTGTACTGTATTCTTATAGTG AAGCCACA-GATGTATAAGAATACAGTACAAAGCT AGTGCCTACTGCCTCGGA-3'; shSmad4, 5'- TGCTG TTGACAGTGAGCGCTGAGAATGCACAATCGCCGGA TAGTGAAGCCACAGATGTATCCGGCGATTGTGCAT TCTCAATGCCTACTGCCTCG - $3^{\prime}$. The vector for shSmad plasmid also has EGFP as a reporter for shRNA expression. The EGFP-shSmad sequences were cloned into the pT3EF1 $\alpha$ vector via the Gateway cloning technology (Invitrogen, Carlsbad, CA). Plasmids were purified using the Endotoxin free Maxi Prep Kit (Sigma-Aldrich, St. Louis, MO, USA).

\section{Mouse treatment and hydrodynamic tail vein gene delivery}

Wild-type $F V B / N$ mice were obtained from Charles River (Wilmington, MA, USA). Mice used for hydrodynamic injection were 5.5- to 6-week-old. Mice were randomly divided in control and experimental groups and numbers of mice in each group are shown in the related figure legends. No blinding was applied in the study. Hydrodynamic tail vein injections ( $\mathrm{HTVi}$ ) were performed as described ${ }^{19}$. Dosages of the plasmids were as follows: cMyc $10 \mu \mathrm{g}$ (or $15 \mu \mathrm{g}$ for shSmad combination), Mcl-1 $10 \mu \mathrm{g}$, TGF $3140 \mu \mathrm{g}$, shLuciferase $30 \mu \mathrm{g}$, shSmad2 $30 \mu \mathrm{g}$, shSmad3 $30 \mu \mathrm{g}$, shSamd4 $30 \mu \mathrm{g}$, pCMV $60 \mu \mathrm{g}$, pCMV-Cre $60 \mu \mathrm{g}$, pT3 $60 \mu \mathrm{g}$. The dosage for sleeping beauty (SB) was $1 / 25$ of the total oncogene injected. A detailed protocol for HTVi can be accessed at https://pharm.ucsf.edu/ xinchen/protocols/hydrodynamic-tail-injection. Mice were monitored by abdominal palpation and euthanized when they developed a high burden of liver tumors, i.e. large abdominal masses. Mice were housed, fed, and monitored in accordance with protocols approved by the Committee for Animal Research at the University of California, San Francisco.

\section{Murine intrasplenic injection induced liver tumor model}

Inducible TGF $\beta 1$ (or EGFP) expressing HCC4-4 cells were delivered into the mouse liver through intrasplenic injection via the splenic vein, which joins with the portal vein, as previously described ${ }^{20}$. Briefly, $100 \mu \mathrm{l}$ ice cold phosphate-buffered saline solution containing $1 \times 10^{6}$ cells was injected to the spleen of 8-week-old $F V B / N$ mouse under general anesthesia. Three days post injection, mice were harvested to evaluate the tumor formation in the spleen at this time point. Doxycycline food (Envigo RMS Inc., Indianapolis, IN) was administrated 3 days after cells implantation. Three mice from each arm (EGFP or TGF $\beta 1$ ) were sacrificed 4 weeks after implantation, and then mice were sacrificed every 3 days, one per group, in parallel. Mouse organs including spleen, liver, lymph node, pancreas, adrenal gland, kidney, colon, diaphragm, abdominal muscles, and lungs were collected for further molecular and histological analyses.

\section{Protein extraction and Western blot analysis}

For total protein extraction, mouse liver tissues and cells were homogenized in M-PER ${ }^{\mathrm{TM}}$ Mammalian Protein Extraction Reagent (Cat\#78501, Thermo Fisher Scientific) containing the Halt ${ }^{\mathrm{TM}}$ Protease Inhibitor Cocktail (Cat\#78429, Thermo Fisher Scientific). Subsequently, protein concentration was determined using the Pierce ${ }^{\mathrm{TM}}$ Microplate BCA Protein Assay Kit (Cat\#23252, Thermo Fisher Scientific). For Western blotting, extracted proteins were boiled in Tris-Glycine SDS Sample Buffer (Bio-Rad Laboratories, Inc., Hercules, CA) for denaturation and 
subsequently separated by SDS-PAGE, and transferred onto nitrocellulose membranes (Bio-Rad Laboratories, Inc.) by electroblotting. Membranes were blocked in $10 \%$ non-fat milk in Tris-buffered saline containing $0.05 \%$ Tween-20 for $1 \mathrm{~h}$ at room temperature and then incubated with primary antibodies (summarized in Supplementary Table 1) at $4{ }^{\circ} \mathrm{C}$ overnight. Membranes were then incubated with horseradish peroxidase-secondary antibody (1:5000; Jackson ImmunoResearch Laboratories Inc., West Grove, PA) at room temperature for $1 \mathrm{~h}$. After appropriate washing, membranes were developed with the Clarity $^{\mathrm{TM}}$ Western ECL Substrate (Cat\#170-5061, BioRad Laboratories, Inc.) or Clarity ${ }^{\mathrm{TM}}$ Max Western ECL Substrate (Cat\#170-5062, Bio-Rad Laboratories, Inc.).

\section{RNA extraction and quantitative real-time polymerase chain reaction (qRT-PCR)}

Total mRNA from mouse liver tissues and cells was extracted by using the Quick RNA miniprep kit (Zymo Research, Irvine, CA, USA). Next, mRNA expression was detected by qRT-PCR using the SYBR Green Master Mix (Applied Biosystems, Foster City, CA, USA) in a QuantStudio $^{\mathrm{TM}} 6$ Flex system (Applied Biosystems). Expression of each gene was normalized to the $18 \mathrm{~S}$ rRNA gene. Thermal cycling started with an initial hold period at $95^{\circ} \mathrm{C}$ for $10 \mathrm{~min}$, and then followed by a three-step PCR program of $95^{\circ} \mathrm{C}$ for $15 \mathrm{~s}, 60^{\circ} \mathrm{C}$ for $1 \mathrm{~min}$, and $72^{\circ} \mathrm{C}$ for $30 \mathrm{~s}$ for a total of 40 cycles. Primers used are listed in Supplementary Table 2.

\section{Cell culture and in vitro studies}

Mouse HCC cell lines (HCC3-4, HCC4-4) were obtained from Dr. Dean Felsher (Stanford University, Palo Alto, CA). The following human HCC cell lines were also used for the in vitro studies: Hep40, HLF, Huh7, and SNU475. Cell lines were authenticated (Genetica DNA Laboratories, Burlington, $\mathrm{NC}$ ) and tested clear of mycoplasma contamination. Cells were cultured in DMEM medium (Gibco, Grand Island, NY, USA) with 5\% fetal bovine serum (Gibco), $100 \mu \mathrm{g} / \mathrm{ml}$ streptomycin, and $100 \mathrm{U} / \mathrm{ml}$ penicillin at $37^{\circ} \mathrm{C}$ in a $5 \% \mathrm{CO}_{2}$ humidified incubator. For transfection of EGFP or TGF $\beta 1$, pCW57.1EGFP or pCW57.1-TGF $\beta 1$ lentivirus was added to the culture medium when cells reached $50 \%$ to $60 \%$ confluency in $60 \times 15 \mathrm{~mm}$ culture dishes. Seventy-two hours later, cells were trypsinized and cultured in $100 \times 20 \mathrm{~mm}$ dishes in culture medium containing puromycin at the concentration of $1.5 \mu \mathrm{g} / \mathrm{ml}$ for SNU475, $2 \mu \mathrm{g} / \mathrm{ml}$ for HCC3-4 and Huh7, $3 \mu \mathrm{g} / \mathrm{ml}$ for HCC4-4, $5 \mu \mathrm{g} / \mathrm{ml}$ for HLF and $15 \mu \mathrm{g} / \mathrm{ml}$ for Hep40. After 3 days of selection, cells were used for cell migration /invasion and colony formation studies. EGFP and TGF- $\beta 1$ expression were induced by Doxycycline at a concentration of $4 \mu \mathrm{g} / \mathrm{ml}$. Proliferation and migration /invasion assays were conducted as described before ${ }^{14}$. For Pmepa1 knockdown, cells were transfected with siScramble or siPmepa 1 by using the Thermo Fischer Scientific siRNA Transfection Kit. Cells were harvested for RNA extraction at $48 \mathrm{~h}$ after transfection.

\section{Histology, immunohistochemistry and proliferation and apoptotic indices}

Histopathologic examination of the mouse lesions was conducted by a board-certified pathologist and liver expert (M.E.), in accordance with the criteria described previously ${ }^{18}$. Antigen unmasking was achieved by placing the slides in a microwave oven on high for 10 min either in $10 \mathrm{mM}$ sodium citrate buffer ( $\mathrm{pH} 6.0)$ or $1 \mathrm{mM}$ ethylenediaminetetraacetic acid (EDTA; $\mathrm{pH}$ 8.5), followed by a 20-min cool down at room temperature. After a blocking step with the $5 \%$ goat serum and Avidin-Biotin blocking kit (Vector Laboratories, Burlingame, CA), the slides were incubated with primary antibodies overnight at $4{ }^{\circ} \mathrm{C}$. Slides were then subjected to $3 \%$ hydrogen peroxide for $10 \mathrm{~min}$ to quench endogenous peroxidase activity and, subsequently, the biotin-conjugated secondary antibody was applied at a 1:500 dilution for $30 \mathrm{~min}$ at room temperature. The antibodies used in the experiments are described in Supplementary Table 4. Immunoreactivity was visualized with the Vectastain Elite ABC kit (Cat\#PK-6100, Vector Laboratories, Burlingame, CA) and ImmPACT $^{\circledR}$ DAB Peroxidase (HRP) Substrate (Cat\#SK-4105, Vector Laboratories, Burlingame, CA). Slides were counterstained with hematoxylin. Proliferation and apoptosis indices were determined in mouse HCC lesions by counting Ki67 and Cleaved Caspase 3 positive cells, respectively. ImageJ 1.8.0 (National Institutes of Health, USA, https://imagej.nih.gov/ij/download. html) and Image Pro Plus 7 (Media Cybernetics, Rockville, $M D$ ) software were used for quantification.

\section{TCGA data analysis}

For c-Myc Oncoprint and co-expression analysis, liver hepatocellular carcinoma (TCGA, PanCancer Atlas) data at the public cBioPortal site were utilized. Samples with MYC mutation data (442 patients/samples) were obtained. Genes in Ensembl ID were converted to Entrez ID using the Bioconductor Package Maintainer org.Hs.eg. $\mathrm{db}$, version 3.8.2. Edge $\mathrm{R}$ package was used for KEGG analysis. Spearman's correlation of PMEPA1 mRNA expression and TGFB1, TGFB2 or TGFB3 mRNA expression was also assessed. For survival analysis, RNAseq data, which contain gene expression information of 50 normal liver samples and $370 \mathrm{HCC}$ patients, were obtained from the TCGA datasets. Updated follow-up survival data were downloaded using the $R$ package TCGAbiolinks. The normalized count data calculated by expectation maximization analysis ${ }^{21}$ were incorporated as 
a matrix in R. With the adoption of an enrichment score cutoff determined by the maximal chi-square method ${ }^{22}$ using R package Maxstat, TCGA HCC samples were categorized into PMEPA1-high and PMEPA1-low signature groups.

\section{Statistical analysis}

The Prism 7.0 software (GraphPad, San Diego, CA) was used to analyze the data, which are presented as Means \pm $\mathrm{SD}$. Comparisons between two groups were performed using the two-tailed unpaired $t$-test. Welch correction was applied when necessary. $P$-values $<0.05$ were considered statistically significant. Kaplan-Meier survival data were evaluated using a log-rank (Mantel-Cox) test.

\section{Results}

\section{Activation of the TGF $\beta$ pathway in c-MYC amplified human HCC samples}

To investigate the genes and pathways regulated by cMYC amplification, we performed bioinformatics analysis of HCC samples from The Cancer Genome Atlas (TCGA) LIHC cohort and identified 2198 genes whose expression levels were deregulated in HCCs harboring c-MYC amplification (Supplementary Table 3). Subsequently, these genes were subjected to Kyoto Encyclopedia of Genes and Genomes (KEGG) analysis. Interestingly, the readout showed a significant TGF $\beta$ signaling cluster in cMYC amplified liver tumors (Fig. 1a). In particular, the expression of TGF $\beta$ target genes, such as $E 2 F 5^{23}$, $R H O A^{24}, R B X 1^{25}$, and $P P P 2 R 1 A^{26}$, was higher in HCC samples with c-MYC amplification (MYC Amp) than those without amplification (MYC Wt) (Fig. 1b).

To further substantiate this observation, we investigated whether TGF $\beta$ signaling is also activated in mouse HCC induced by hydrodynamic tail vein injection ( $\mathrm{HTVi}$ ) of the c-Myc protooncogene ${ }^{14}$. Levels of c-Myc, activated/ phosphorylated forms of Smad2/3 (p-Smad2/3) and total Smad2/3 were assessed by Western blot. Upregulation of p-Samd $2 / 3$ was found in c-Myc mouse tumors, indicating the activation of the TGF $\beta$ signaling (Fig. 1c). Moreover, microarray analysis of $\mathrm{c}-\mathrm{Myc}$ mouse tumor lesions ${ }^{14}$ revealed significant elevation of TGF $\beta$ target genes, including E2f5, Rhoa, Rbxl, and Ppp2rla in mouse HCC (Fig. 1d).

In summary, our data indicate the activation of the TGF $\beta$ pathway in c-MYC induced HCC in humans and mice, suggesting a possible functional crosstalk between TGF $\beta$ and c-MYC cascades during hepatocarcinogenesis.

\section{Activation of the TGF $\beta / S M A D$ signaling delays HCC development driven by c-Myc overexpression}

Previous studies suggest that the TGF $\beta$ signaling might act either as tumor promoter or tumor suppressor in many cancer types, including $\mathrm{HCC}^{10,15}$. As a first step to elucidate the mechanisms whereby TGF $\beta$ modulates cMYC driven HCC development, we determined whether activation of TGF $\beta$ accelerates or delays c-Myc HCC initiation in vivo. For this purpose, we co-expressed the TGF $\beta 1$ plasmid together with the c-Myc oncogene (cMyc/TGF $\beta 1$ ) via HTVi. The control group was injected with c-Myc and pT3-EF1 $\alpha$ empty vector plasmids (c-Myc/ pT3; Fig. 2a). We found that mice in control group rapidly developed HCCs and were moribund by 5 weeks after injection, whereas no preneoplastic and neoplastic lesions were observed in c-Myc/TGF $\beta 1$ injected murine livers at the same time point (Fig. 2b). The c-Myc/pT3 HCC lesions demonstrated significant higher proliferation rate than c-Myc/TGF $\beta 1$ livers, as determined by anti-Ki67 immunohistochemistry (Fig. 2c, d). Over long time, overexpression of TGF $\beta 1$ led to a significant increased overall survival in c-Myc injected mice (Fig. 2e). A few small tumor nodules were observed in c-Myc/TGF $\beta 1$ livers at 11.6 weeks post injection, and several larger individual tumor lesions were detected by 18 weeks post injection. Nonetheless, the number of tumor nodules was limited in c-Myc/TGF $\beta 1$ mice (Fig. 2f). Moreover, c-Myc/ TGF $\beta 1$ HCCs exhibited both active proliferation and apoptotic cell death, as indicated by Ki67(+) cells and cleaved caspase-3 (C-C3) (+) cells (Fig. 2f). As expected, the levels of $\mathrm{p}-\mathrm{Smad} 2 / 3$ were higher in $\mathrm{c}-\mathrm{Myc} / \mathrm{TGF} \beta 1$ tumors than c-Myc/pT3 corresponding lesions (Supplementary Fig. 1), indicating the strong activation of TGF $\beta$ signaling in $\mathrm{c}-\mathrm{Myc} / \mathrm{TGF} \beta 1$ mouse liver tumors.

Next, we investigated whether TGF $\beta 1$ dependent suppression of c-Myc driven hepatocarcinogenesis was mediated by the Smad2/3 and Smad 4 complexes. Thus, we generated short hairpin RNAs (shRNAs) targeting Smad2, Smad3, and Smad4 into pT3-EF1 $\alpha$ vector downstream of the GFP sequence. Subsequently, shSmad2, shSmad3, or shSmad4 plasmids were coexpressed with c-Myc in the mouse liver via HTVi. Additional mice were injected with c-Myc together with shRNA against Luciferase (shLuciferase) as control (Fig. 3a). Importantly, silencing of Smad2, Smad3 or Smad4 significantly accelerated c-Myc HCC formation (Fig. 3b). By 5 weeks after injection, tumor nodules were obvious in shSmad2, shSmad3, and shSamd4 co-injected mice, while neoplastic lesions were rarely detected in control shLuciferase injected mice (Fig. 3c). It is worth to note that while silencing of either Smad2 or Smad3 led to accelerated c-Myc tumor growth, silencing of Smad4 demonstrated the most significant tumor acceleration phenotype (Fig. 3b and 3c). This observation is consistent with the fact that Smad2 and Smad3 may be functional redundant, whereas Smad4 is the unique subunit in the Smad complex downstream of TGF $\beta$ signaling ${ }^{27}$. Histologically, a small cluster of HCC tumor cells with lower proliferation rate, as indicated by Ki67 staining, was 


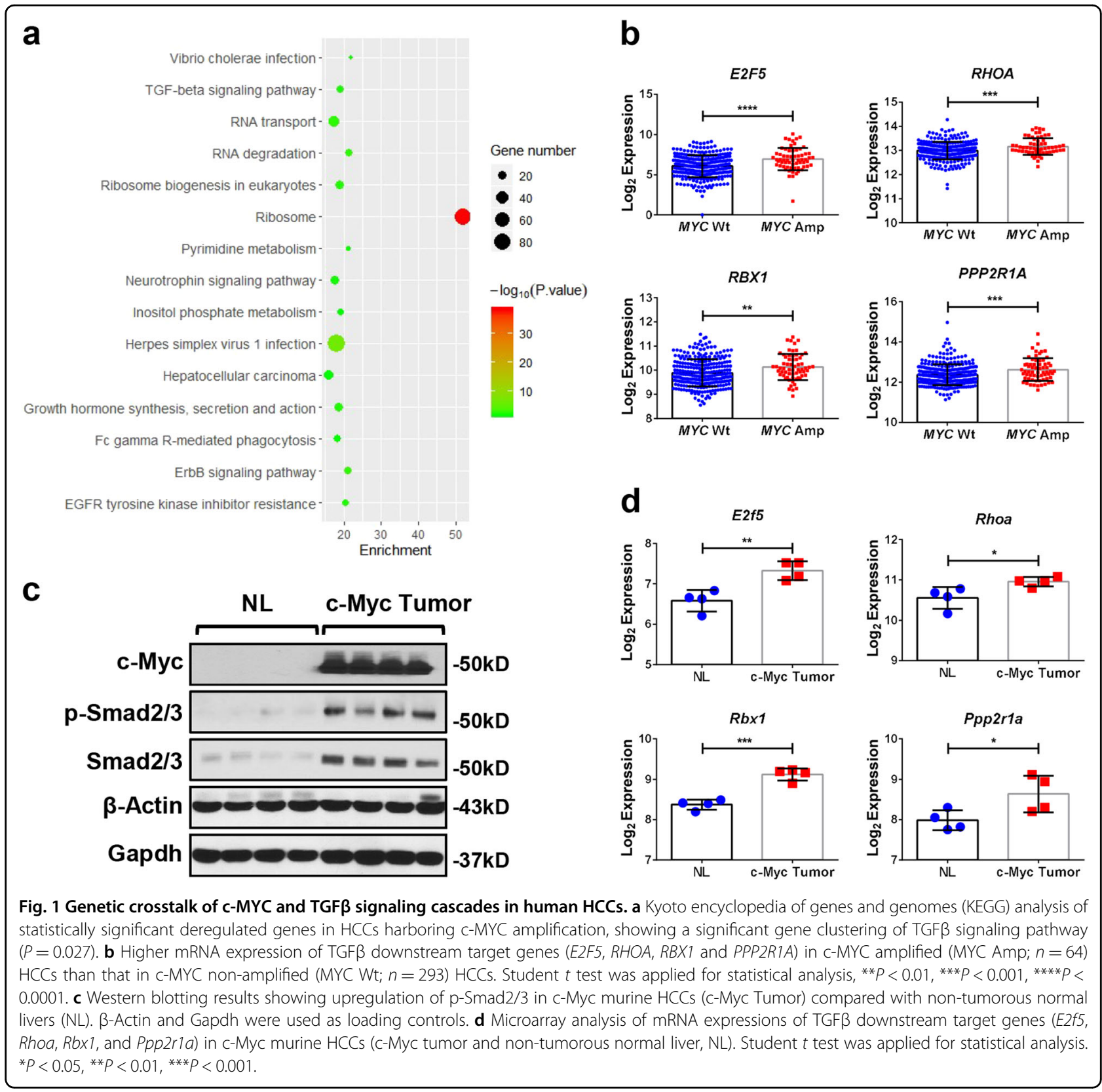

detected in shLuciferase injected mice (Supplementary Fig. 2a). The expression of shSmads or shLuciferase in HCC lesions was validated via immunofluorescence staining of GFP (Supplementary Fig. 2b). Altogether, the present data indicate that silencing of Smad2/3/4 accelerates c-Myc driven $\mathrm{HCC}$ initiation.

Next, we investigated the molecular mechanisms underlying the observed phenotypes. Thus, we analyzed tumor cell proliferation and apoptosis rates in c-Myc/ shLuciferase and c-Myc/shSmads mouse HCC. Intriguingly, cell proliferation showed no difference between cMyc/shLuciferase and c-Myc/shSmads liver tumors, as indicated by Ki67 index (Supplementary Fig. 2c).
In contrast, cell apoptosis was dramatically hampered in shSmad2, shSmad3, and shSmad4 HCC lesions, as measured by cleaved caspase 3 index (Fig. $3 \mathrm{~d}$ and e).

Altogether, these findings indicate that the TGF $\beta$ pathway might modulate c-Myc HCC initiation by regulating c-Myc induced apoptosis. Specifically, inhibition of TGF $\beta$ might prevent c-Myc induced cell death, leading to accelerated tumor development, whereas overexpression of TGF $\beta 1$ might facilitate apoptosis, resulting in the delay of HCC formation. If this hypothesis is correct, one could predict that co-expression of an anti-apoptotic mediator, such as Mcl-1, would rescue TGF $\beta 1$ dependent tumor inhibition phenotype, i.e., overexpression of TGF $\beta 1$ would not be able 

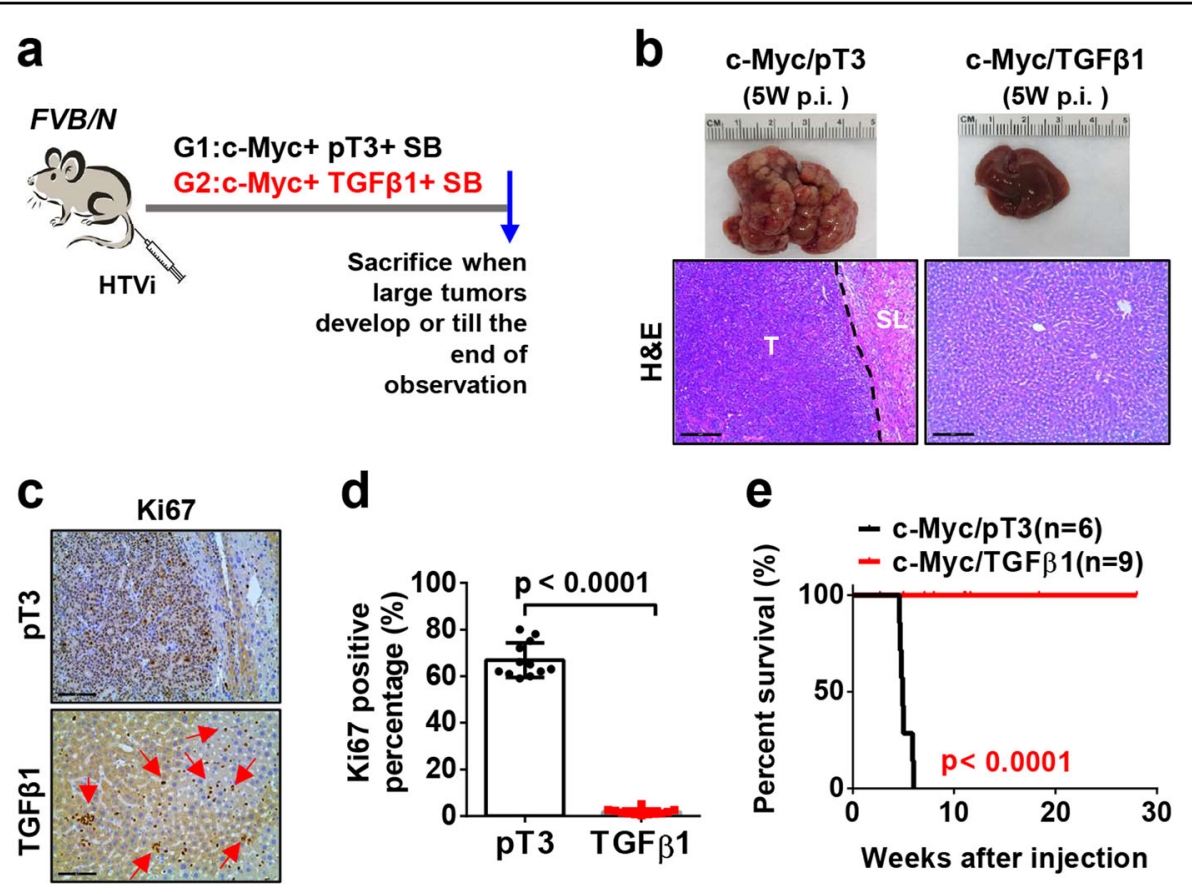

f

5W

11.6W

18.4W
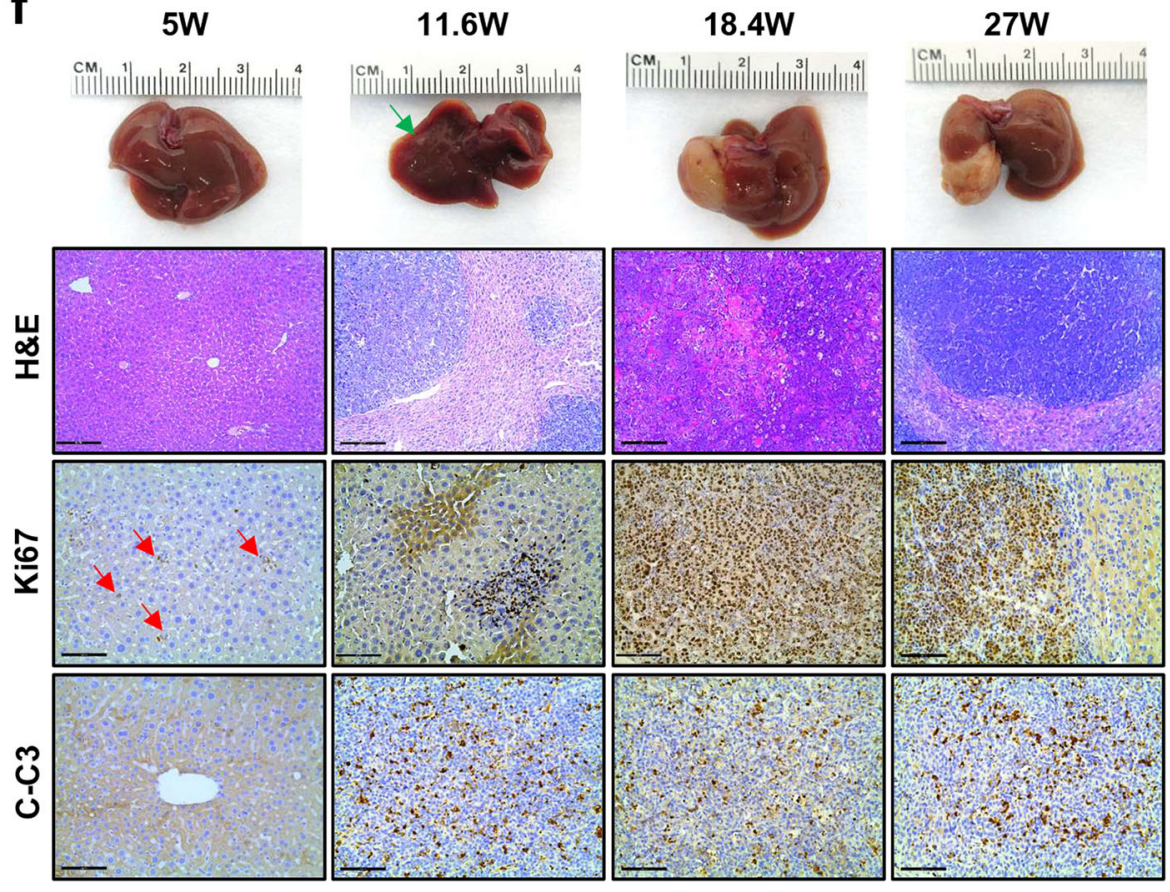

Fig. 2 Activation of TGF $\beta 1$ delays c-Myc induced hepatocarcinogenesis in mice. a Study design. FVB/N mice were injected with c-Myc/pT3/SB $(N=6)$ or $c-M y c / T G F B 1 / S B(N=9)$ plasmids. Mice were monitored and sacrificed when moribund. b Representative images of tumor and H\&E

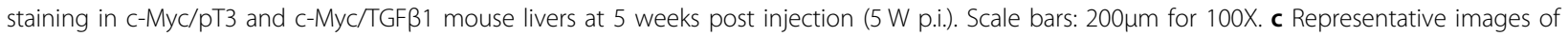
Ki67 staining in c-Myc/ pT3 and c-Myc/TGF 31 mouse livers at 5 weeks post injection ( $5 \mathrm{~W}$ p.i.). Red arrows indicate Ki67+ cells in c-Myc/TGF $\beta 1$ group. Scale bars: $100 \mu \mathrm{m}$ for 200X. d Quantification of Ki67+ cells percentages in in c-Myc/pT3 and c-Myc/TGF $\beta 1$ mouse livers. Student $t$ test was applied for statistical analysis. e Survival curve showing that TGF $\beta 1$ impaired c-Myc induced hepatocarcinogenesis in mice. Kaplan-Meier method and logrank test were applied. $P<0.0001$. f Representative images of liver, H\&E, Ki67, and Cleaved Caspase-3 (C-C3) immunohistochemical staining in c-Myc/ TGF $\beta 1$ group at different time points after injection. Green arrow indicates a tumor nodule on the surface of the right lobe of mouse liver. Red arrows indicate Ki67+ cells. Scale bars: $200 \mu \mathrm{m}$ for H\&E, $100 \mu \mathrm{m}$ for Ki67 and C-C3. 

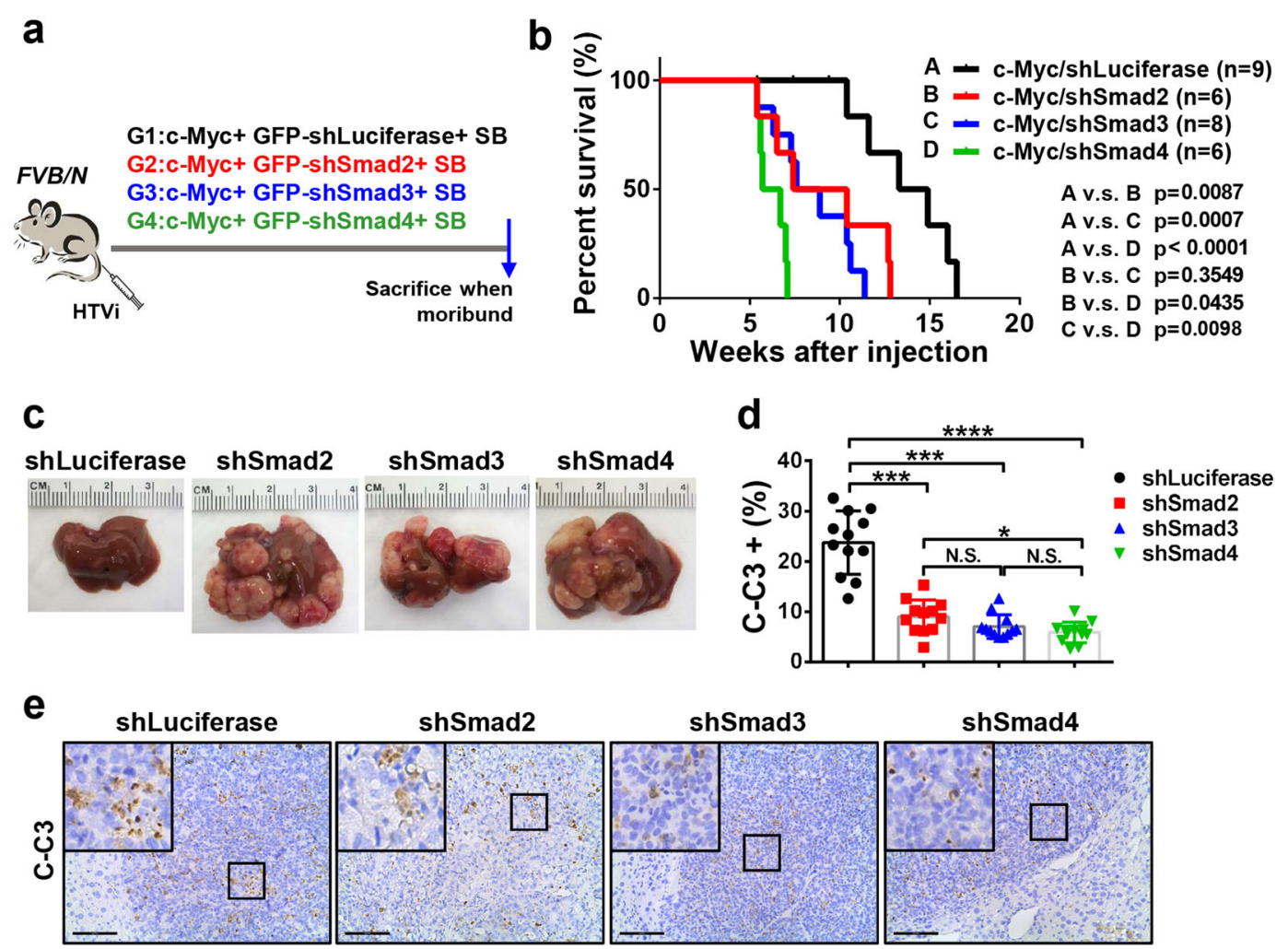

Fig. 3 Inhibition of TGF $\beta$-SMAD signaling pathway accelerates c-Myc liver tumorigenesis in mice. a Study design. FVB/N mice were hydrodynamically injected (HTVi) with c-Myc/GFP-shLuciferase/SB $(n=9)$, c-Myc/GFP-shSmad2/SB $(n=6)$, c-Myc/GFP-shSmad3/SB $(n=8)$ and cMyc/GFP-shSmad4/SB $(n=6)$ plasmids, respectively. Mice were monitored and sacrificed when moribund. $\mathbf{b}$ Survival curve showing that inhibition of Smad2, Smad3 or Smad4 accelerates c-Myc induced hepatocarcinogenesis in mice. Kaplan-Meier method and log-rank test were applied. $P$-values were shown in the figure. $\mathbf{c}$ Representative images of tumors in the four groups at 5 weeks post injection (p.i.). $\mathbf{d}$ Comparison of cleaved caspase 3 (CC3) positive cells percentages in the four groups. Student $t$-test was applied between each 2 groups for statistical analysis, ${ }^{*} P<0.05$, ${ }^{* * *} P<0.001$, ${ }^{* * * * P}<0.0001$, N.S., no significance. e Representative images of cleaved caspase 3 (C-C3) immunohistochemical staining in c-Myc/shLuciferase (shLuciferase), c-Myc/shSmad2 (shSmad2), c-Myc/shSmad3 (shSmad3) and c-Myc/shSmad4 (shSmad4) mouse HCCs. Scale bars: 200 m.

to suppress tumor development induced by c-Myc and Mcl1 oncogenes in the liver. To test the hypothesis, we codelivered c-Myc, Mcl-1, and TGF $\beta 1$ (c-Myc/Mcl-1/TGF $\beta 1$ ) plasmids to the mouse liver. Additional mice were injected with c-Myc, Mcl-1, and pT3-EF1 $\alpha$ empty vector (c-Myc/ Mcl-1/pT3) as control (Supplementary Fig. 3a). Noticeably, consistent with our hypothesis, overexpression of TGF $\beta 1$ did not affect liver tumor development in $\mathrm{c}-\mathrm{Myc} / \mathrm{Mcl}-1$ mice (Supplementary Fig. 3b-d).

In summary, the present data show that overexpression of TGF $\beta 1$ significantly delays c-Myc dependent hepatocarcinogenesis, whereas suppression of the TGF $\beta / \mathrm{Smad}$ pathway accelerates c-Myc HCC initiation by hindering cMyc induced apoptosis.

\section{TGF $\beta 1$ promotes c-Myc liver tumor metastasis}

Our study points to a tumor suppressor role of the TGF $\beta /$ Smad cascade during tumor initiation. Since the TGF $\beta$ pathway possesses context-dependent tumor inhibitory and tumor promoting activities ${ }^{10,15}$, we sought to investigate the role of TGF $\beta$ on c-Myc driven tumor progression. For this purpose, we applied murine HCC cell lines, namely HCC3-4 and HCC4-4 cells, which are derived from c-Myc mouse $\mathrm{HCCs}^{28}$. Specifically, HCC3-4 and HCC4-4 cell lines were transfected either with the doxycycline inducible TGF $\beta 1$ expression plasmid or with the EGFP construct (as control). As expected, following doxycycline administration, HCC3-4 and HCC4-4 cells exhibited higher levels of $\mathrm{p}-\mathrm{Smad} 2 / 3$ when transfected with TGF $\beta 1$, indicating the activation of TGF $\beta$-Smad signaling upon TGF $\beta 1$ overexpression (Fig. 4a). Activation of the TGF $\beta$ pathway did not affect c-Myc HCC cell growth, as determined by cell proliferation and colony formation assays (Supplementary Fig. 4). In contrast, activation of the TGF $\beta$ signaling significantly promoted cMyc tumor cell migration, as measured by cell wound healing assay (Fig. 4b, c), and cell invasion, as evaluated by transwell migration assay (Fig. 4d, e). 


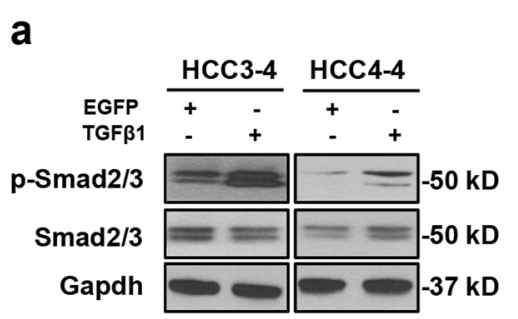

C

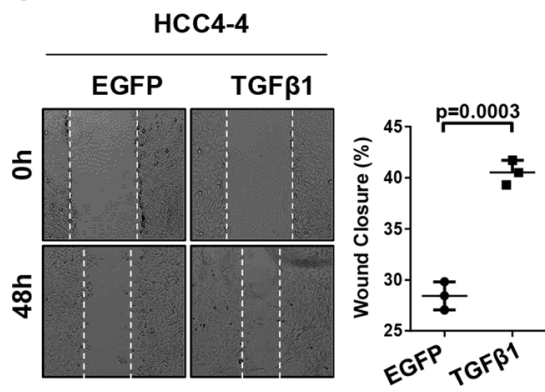

b

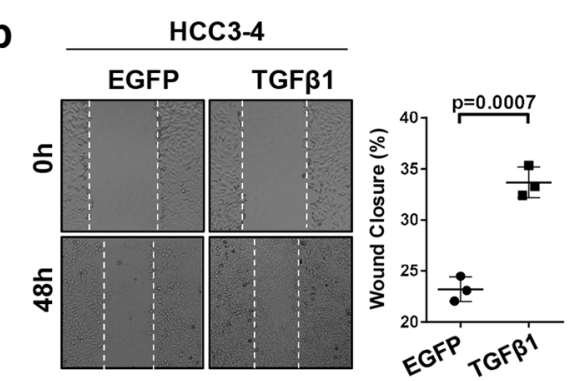

d
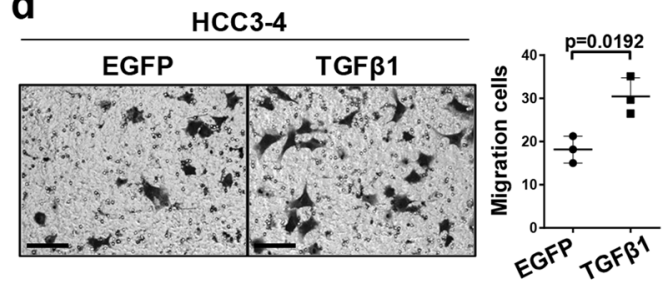

e
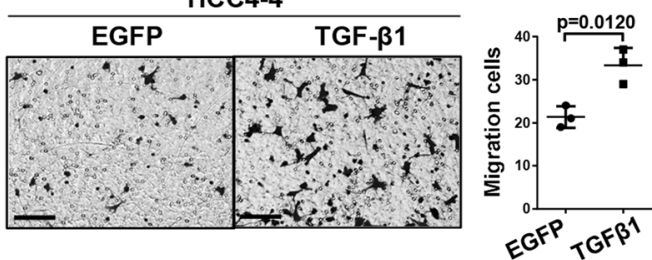

Fig. 4 TGF $\beta 1$ activation promotes cell migration and invasion in c-Myc murine tumor derived HCC cell lines. a Western blotting results showing activation of Smad2/3 ( $\mathrm{p}-\mathrm{Smad} 2 / 3$ ) in TGF 31 overexpressing HCC3-4 and HCC4-4 cells. Gapdh was used as a loading control. b, c Representative images and quantifications of cell healing assay at 0-hour ( $0 \mathrm{~h}$ ) and after 48 hours $(48 \mathrm{~h})$ treatment for inducing TGF $\beta 1$ ( $N=3$ ) or EGFP $(N=3)$ expression in $\mathbf{b}$ HCC3-4 and $\mathbf{c}$ HCC4-4 cell lines. $\mathbf{d}$, e Representative images and quantification of transwell migration assay $48 \mathrm{~h}$ after treatment for inducing TGF $31(N=3)$ or EGFP $(N=3)$ expression in (d) HCC3-4 and (e) HCC4-4 cell lines. Student $t$ test was applied for statistical analysis. $P$ values were as shown. Experiments were conducted three times.

Next, to delineate the role of TGF $\beta 1$ signaling in modulating the progression, especially metastasis, of cMyc induced mouse HCCs in vivo, we employed the intrasplenic injection tumor model. Specifically, inducible TGF $\beta 1$ (or EGFP) expressing HCC4-4 cells were delivered into the mouse liver through intrasplenic injection via the splenic vein, which joins with the superior mesenteric vein to become the portal vein ${ }^{20}$. It is important to note that, consistent with previous findings ${ }^{29}$, microscopic lesions could be clearly visualized in the mouse spleen by 3 days after injection (Supplementary Fig. 5), suggesting that the tumors have developed at this time point. By administering doxycycline with the food, TGF $\beta 1$ expression is induced in the mouse HCC, thus allowing the investigation of TGF $\beta 1$ role in tumor progression. Three mice from each arm (EGFP or TGF $\beta 1$ ) were sacrificed 4 weeks after implantation as baseline. Mice were subsequently sacrificed every 3 days, one per group, in parallel (Fig. 5a). Mouse organs, including spleen, liver, lymph nodes, pancreas, adrenal glands, kidney, colon, diaphragm, abdominal muscles, and lungs were collected and subjected to $H \& E$ staining for tumorigenesis analysis. As expected, all mice eventually developed tumors in the spleen, and no difference in terms of tumor burden on the spleen was noted. The results were consistent with in vitro analyses showing that overexpression of TGF $\beta 1$ does not significantly affect tumor growth. Noticeably, TGF $\beta 1$ activation promoted HCC metastasis, as indicated by higher tumor incidence in the liver, abdominal lymph nodes as well as other organs (Fig. 5b). Of note, liver tumor nodules were detected as early as 4 weeks after tumor cell implantation in the TGF $\beta 1$ group, whereas no tumor was detected in the control group. Presence of c-Myc positive tumor cells was confirmed by immunohistochemistry for c-Myc protein (Fig. 5c). Further analysis revealed that the proliferation rate was significantly increased in livers with activated TGF $\beta 1$, as indicated by a higher Ki67(+) cell percentage (Fig. 5d).

Overall, our data show that conditional activation of TGF $\beta 1$ in c-Myc positive HCC cells contributes to tumor metastasis in vitro and in vivo. 

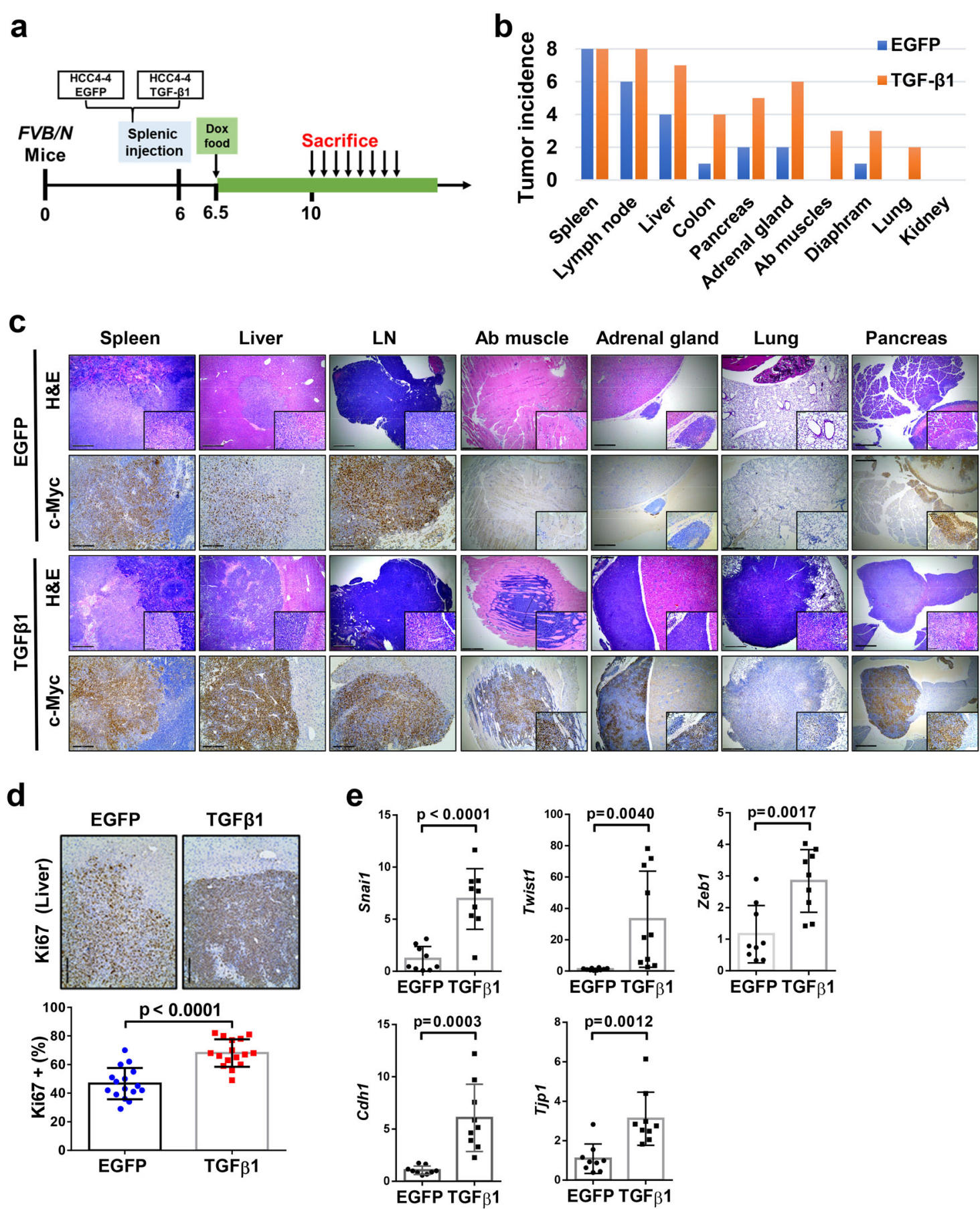

Fig. 5 TGF $\beta 1$ activation promotes c-Myc positive tumor cells progression and metastasis in vivo. a Study design. Inducible TGF $\beta 1$ (or EGFP) expressing HCC4-4 cells were delivered into mouse liver through intrasplenic injection. Food containing doxycycline (Dox food) was administered 3 days after implantation to induce TGF $\beta 1$ or EGFP expression. Three mice from each arm (EGFP or TGF $\beta 1$ ) were sacrificed 4 weeks after implantation, and then mice were sacrificed every 3 days one per group in parallel. $\mathbf{b}$ Numbers of mice showing tumors in each organ at the time of sacrifice from EGFP $(N=8)$ and TGF $\beta 1(N=8)$ groups. The $Y$ axis (tumor incidence) represents the number of mice from the EGFP or TGF $\beta 1$ group displaying neoplastic lesions in each organ. Tumors were examined macroscopically and microscopically by H\&E staining. c Representative images of H\&E and c-Myc immunohistochemical staining in EGFP and TGF $\beta 1$ groups in different organs. Black boxes on right lower corner denote enlarged views for better visualization. Scale bars: 200 mm for H\&E, 100 m for c-Myc. d Representative images of Ki67 immunohistochemical staining and quantification of Ki67 positive cell percentage in liver tumors from both groups. Student $t$ test was applied for statistical analysis. $P<0.0001$. Scale bars: $100 \mu m$. e mRNA expression of TGF $\beta$ downstream target genes (Snai1 Twist1, and Zeb1) and epithelial marker genes (Cdh1 and Tjp1) in EGFP or TGF 31 activated murine liver tumors. Student $t$-test was applied for statistical analysis. 


\section{EMT might be dispensable for TGF $\beta$ to promote c-Myc HCC metastasis}

Next, we focused on the liver lesions induced by the intrasplenic injection method to further explore the function of the TGF $\beta 1$ cascade in c-Myc HCC progression, especially metastasis. Previously, it has been reported that the TGF $\beta$ signaling induces tumor progression by regulating epithelial to mesenchymal transition (EMT) ${ }^{30,31}$ and/or modulating the tumor microenvironment (TME) ${ }^{32,33}$. First, we tested the hypothesis that TGF $\beta$ promotes c-Myc HCC progression and metastasis by inducing EMT. At the molecular level, TGF $\beta$ downstream target genes (Snail, Twist1, and $Z e b 1)$ were found to be upregulated in the lesions. Nonetheless, overexpression of TGF $\beta 1$ led also to the upregulation of epithelial genes (Cdh1 and Tjp1) (Fig. 5e). A similar mRNA trend upon TGF $\beta 1$ overexpression was also observed in vitro using HCC3-4 and HCC4-4 cell lines (Supplementary Fig. 6). Consistently, using immunofluorescence, no overlapping staining pattern for E-cadherin and Vimentin in c-Myc/Mcl-1/TGF 31 murine HCCs was detected (Supplementary Fig. 7), which further substantiated the absence of EMT in TGF $\beta 1$ activated c-Myc tumors.

Next, we investigated whether TGF $\beta$ may modulate EMT in human HCC samples. We reasoned that loss of epithelial cell marker expression was a better indicator than the gain of expression of mesenchymal markers in bulk RNASeq datasets, because one could not distinguish whether the increased mesenchymal marker expression is due to increased fibroblast cells within tumor samples or EMT. We searched TCGA LIHC dataset and analyzed whether TGF $\beta$ ligand isoforms, including TGF $\beta 1$, TGF $\beta 2$, and TGF $\beta 3$, demonstrate a negative correlation with epithelial markers, such as CDH1 and TJP1. We did not observe any negative correlation between TGF $\beta$ isoforms and CDH1 or TJP1 mRNA expression in TCGA LIHC samples (Supplementary Fig. 8).

Altogether, the present findings suggest that TGF $\beta$ induced c-Myc murine HCC metastasis might be independent of the EMT program.

\section{TGF $\beta$ induced TME adaptiveness is required for c-Myc mouse HCC progression}

Previous studies have shown that TGF $\beta$ may modulate TME and promote pro-inflammatory responses ${ }^{34,35}$. To test whether these events might contribute to TGF $\beta$ induced accelerated c-Myc HCC progression, we first examined the changes of tumor related immune milieu in EGFP and TGF $\beta 1$ expressing c-Myc liver tumors. Thus, immunohistochemical analysis for the lymphocyte marker $\mathrm{Cd} 45$ and the macrophage marker F4/80 was performed. Intriguingly, TGF $\beta 1$ activation enhanced lymphocyte infiltration while reduced macrophages in c-Myc liver tumors (Supplementary Fig. 9). These results suggest that TGF $\beta 1$ might be involved in the regulation of the tumor microenvironment in the c-Myc murine model.
Subsequently, we performed quantitative real-time PCR analysis of c-Myc HCC tissues with or without TGF $\beta 1$ overexpression both in vivo and in vitro. Intriguingly, we discovered that TGF $\beta 1$ activated c-Myc murine liver tumors exhibited the upregulation of pro-inflammatory cytokine genes (Interleukin 6, Il-6; and Interleukin 11, Il11), chemokine related genes (chemokine receptor type 4, Cxcr4), and genes associated with tumor microenvironment (parathyroid hormone-related protein, Pthrp; angiopoietin-like 4, Angptl4; transmembrane prostate androgen-induced protein 1, Pmepa1; chloride intracellular channel 4, Clic4; and Jagged-1, Jag-1) (Fig. 6a). Consistently, activation of the TGF $\beta$ signaling also significantly activated the expression of these genes in vitro (Fig. 6b, c).

Among the genes induced by TGF $\beta 1$, transmembrane prostate androgen-induced protein 1 (Tmepa1 or Pmepa1) has been reported to be a biomarker for the TGF $\beta$ inhibitor Galunisertib in $\mathrm{HCC}^{36}$. Thus, we tested whether blocking Pmepal was able to modulate HCC progression. Knocking down of mouse Pmepa1 was achieved by specific small interfering RNAs, and siRNA targeting efficacy was first validated by qRT-PCR (Fig. 6d). Functional analysis revealed that cell migration and invasion were significantly inhibited when knocking down Pmepa 1 in TGF $\beta$ activated HCC3-4 and HCC4-4 cell lines (Fig. 6e-h).

In human HCCs, PMEPA1 mRNA level was found to be significantly correlated with TGF $\beta 1, T G F \beta 2$, and TGF 33 mRNA expression (Fig. $7 \mathrm{a}-\mathrm{c}$ ), further suggesting the tight correlation between TGF $\beta$ signaling and PMEPA1 during liver carcinogenesis. High PMEPA1 expression was also associated with a poor survival outcome (Fig. 7d). Furthermore, $P M E P A 1$ and $c-M Y C$ together might also serve as prognosis marker for HCC, as $c-M Y C$ amplification HCCs with high PMEPA1 expression showed the worst overall survival outcome (Fig. 7e). Furthermore, we found that TGF $\beta 1$ induced PMEPA1 upregulation in $\mathrm{c}-\mathrm{MYC}$ high expressed human HCC cell lines (Hep40 and HLF) ${ }^{37}$, but not in c-MYC low expressed human HCC cell lines (Huh7 and SNU475; Fig. 7f-h), suggesting that PMEPA1 may be a target of TGF $\beta 1$ in the context of c-MYC HCC.

Altogether, the present data indicate a crucial contribution of TGF $\beta 1$ related tumor microenvironment reprogramming in modulating c-Myc HCC progression. PMEPA1 might represent one of the relevant targets induced by TGF $\beta 1$ during this process.

\section{Discussion}

HCC frequently occurs after liver chronic damage in a multistep process, starting from hepatocyte compensatory proliferation to the sequential formation of dysplastic nodules, early tumors, and progressed HCC. The TGF $\beta$ pathway has been reported to be involved in both HCC 


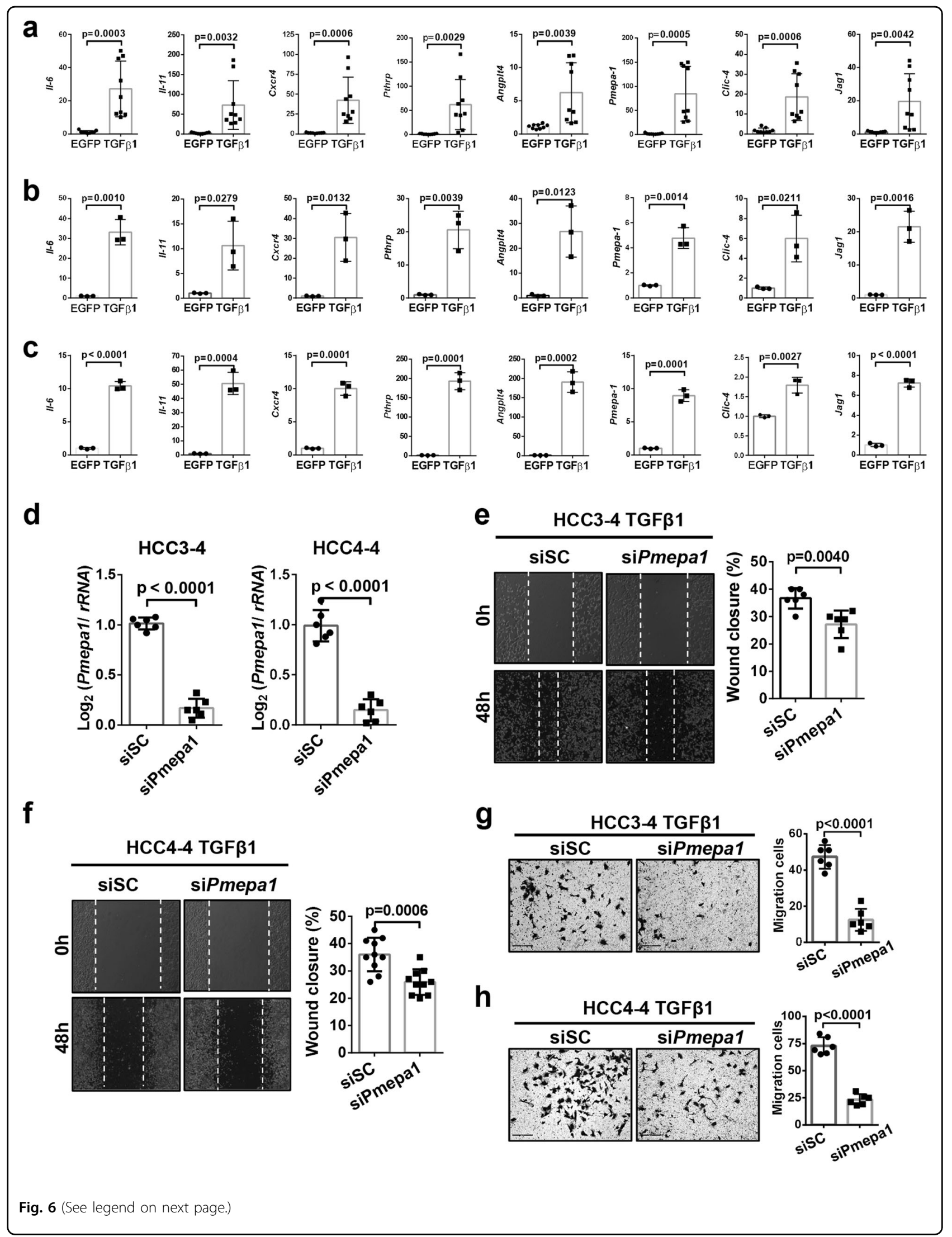


(see figure on previous page)

Fig. 6 TGF $\beta 1$ activation induces tumor microenvironment (TME) reprogramming in c-Myc murine HCCs. a Upregulation of TGF $\beta$ downstream target genes related to TME reprogramming in EGFP or TGF $\beta 1$ activated liver tumors. Student $t$ test was applied for statistical analysis.

b, $\mathbf{c}$ Upregulation of TGF $\beta$ downstream target genes related to TME in EGFP or TGF $\beta 1$ activated $\mathbf{b}$ HCC $3-4$ cell lines and $\mathbf{c}$ HCC4-4 cell lines. Student $t$ test was applied for statistical analysis. $\mathbf{d}$ Quantitative RT-PCR results showing downregulation of Pmepa1 in TGF $\beta 1$ activated HCC3-4 and HCC4-4 cell lines after siPmepa 1 transfection ( $N=6$ replicates in each group). Student $t$ test was applied for statistical analysis. e, $\mathbf{f}$ Representative images and quantification of cell healing assay at 0-hour $(0 \mathrm{~h})$ and after 48 hours $(48 \mathrm{~h})$ treatment following TGF $\beta 1$ overexpression as well as scrambled siRNA (siSC) or siPmepa 1 transfection. Student $t$-test was applied for statistical analysis. P-values were as shown. Experiments were conducted three times. $\mathbf{g}, \mathbf{h}$ Representative images and quantification of transwell assay $48 \mathrm{~h}$ after treatment following TGF $\beta 1$ overexpression as well as scrambled siRNA (siSC) or siPmepa1 transfection. Student $t$-test was applied for statistical analysis. P-values were as shown. Experiments were conducted three times.

initiation and progression stages ${ }^{38,39}$, and members of this cascade have been shown to be mutated in $\sim 40 \% \mathrm{HCC}$ cases $^{6}$. Moreover, the mRNA levels of TGF $\beta$ as well as its downstream effectors, the SMAD family members, are frequently overexpressed in HCC (Supplementary Fig. 10). Nevertheless, the role of the TGF $\beta$ signaling during HCC development remains controversial and the complex function of TGF $\beta$ signaling might be context- and oncogene-dependent ${ }^{40}$. Amplification of c-MYC occurs in a significant subset of human HCCs, and the functional interplay of c-MYC with the TGF $\beta$ signaling remains undefined. Thus, our study provides for the first time a comprehensive investigation of TGF $\beta 1$ during c-MYC liver tumor development and progression.

A major finding of the current study is that TGF $\beta 1$ suppresses HCC initiation in the context of c-Myc activation (Fig. 8). By using hydrodynamic transfection approaches, we provided comprehensive perception of the role of TGF $\beta 1 / S M A D$ pathway in regulating HCC initiation in vivo. Specifically, overexpression of TGF $\beta 1$ hampers hepatocarcinogenesis driven by c-Myc. Consistently, shRNA mediated silencing of Smad2, Smad3, or Smad4, or overexpression of inhibitory $\operatorname{Smad} 7$ (data not shown) led to accelerated $\mathrm{HCC}$ onset in c-Myc mice. Of note, the mixture of $\mathrm{c}-\mathrm{Myc}$ and shRNA expressing plasmids induced murine $\mathrm{HCC}$ later than $\mathrm{c}-\mathrm{Myc}$ in combination with other plasmids. This event might be due to the high diluting efficiency of shRNA plasmids and the high cell death level induced by shRNA expressed plasmid. Our finding is consistent with the previous study by Moon et al., showing that TGF $\beta$ inhibition promotes RAS mediated oncogenesis in c-Myc overexpressing livers ${ }^{17}$. Mechanistically, we showed that activation of the TGF $\beta$ cascade triggers $\mathrm{c}-\mathrm{Myc}$ induced apoptosis, leading to the suppression of tumor initiation. Indeed, when $c-M Y C$ activation/overexpression was coupled to overexpression of the anti-apoptotic gene $\mathrm{Mcl}-1$, the pro-apoptotic function of TGF $\beta 1$ was bypassed, ultimately resulting in tumor formation.

It is worth to note that tumor lesions, although with a longer latency, still developed in c-Myc/TGF $\beta 1$ injected mice. Nonetheless, in contrast to the high number of
$\mathrm{HCC}$ lesions in the c-Myc HCC model, tumor number in c-Myc/TGF $\beta 1$ mice was extremely low. Intriguingly, the eventual liver tumor nodules developing in c-Myc/TGF $\beta 1$ mice expressed higher levels of multiple anti-apoptosis molecules, including B-cell lymphoma-extra-large (Bcl-xl) and Mcl-1 (Supplementary Fig. 11). Considering the limited tumor nodules in c-Myc/TGF $\beta 1$ HCC model, one plausible explanation is that a small percentage of $\mathrm{c}-\mathrm{Myc}$ injected hepatocytes were able to induce the expression of these anti-apoptotic genes, thus escaping TGF $\beta 1$ induced apoptosis. Clearly, additional studies are required to investigate the precise mechanisms whereby $\mathrm{c}-\mathrm{Myc}$ overexpressing hepatocytes induce the expression of these anti-apoptotic genes, and whether this phenotype depends on secondary mutations occurring in tumor nodules or due to additional epigenetic modifications.

Another finding of our investigation is that TGF $\beta 1$ modulates c-Myc HCC metastasis. The studies were performed using murine c-Myc derived HCC cell lines, and both in vitro migration/invasion and in vivo metastasis assays were conducted. An alternative approach to the usage of cell lines would consist in the direct induction of TGF $\beta 1$ in c-Myc HCC mouse lesions. This could be achieved via generating the pT3-TRETGF $\beta 1$ plasmid, and injecting the plasmid into mice together with $\mathrm{c}-\mathrm{Myc}$ and sleeping beauty transposase constructs via HTVi into rtTA transgenic mice ${ }^{41}$. Subsequently, mice would be allowed to develop tumors, and TGF $\beta 1$ could be induced in tumor bearing mice via feeding the mice a doxycycline diet. This approach would provide further support for the role of TGF $\beta 1$ in regulating c-Myc HCC progression in vivo. Importantly, such approach will be necessary to investigate TGF $\beta 1$ in regulating tumor progression when $\mathrm{HCC}$ cell lines are not available, such as in $c$-Met/ $\beta$-Catenin induced mouse HCC.

Mechanistically, we found that TGF $\beta 1$ regulates tumor progression via tumor microenvironment reprogramming rather than by inducing EMT. Although previous studies suggested that EMT is a major cellular mechanisms in TGF $\beta$ pro-tumorigenic function ${ }^{42}$, our results show the lack of EMT induction in TGF $\beta 1$ activated c-Myc HCCs 


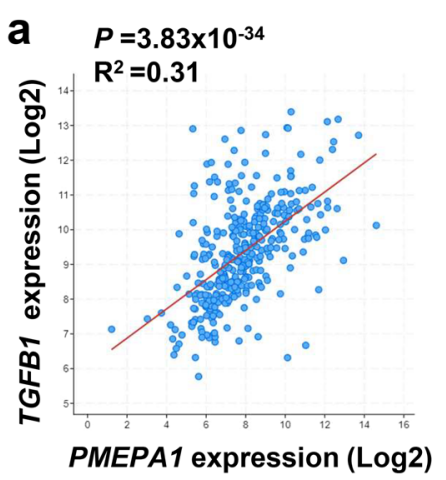

d

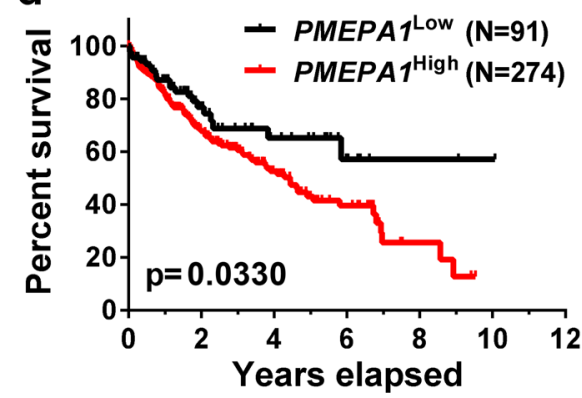

$P=3.23 \times 10^{-25}$

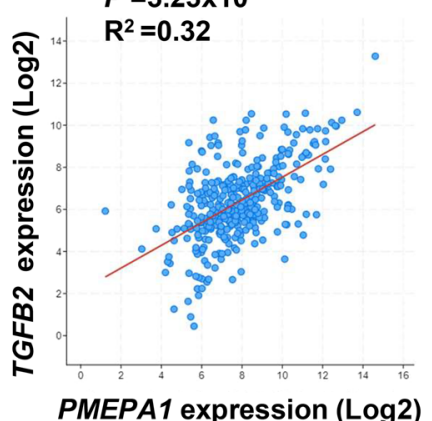

e

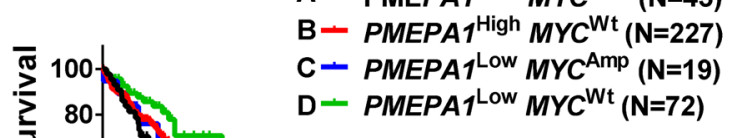

f

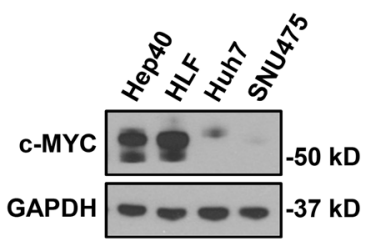

g
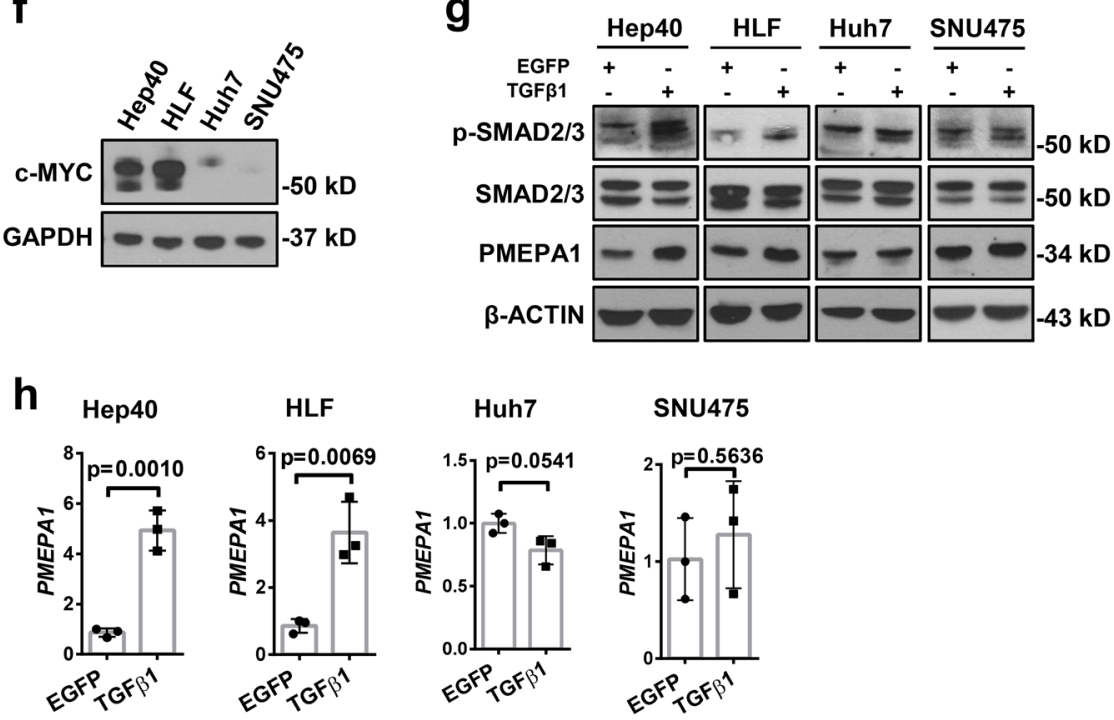

HLF

Huh7

SNU475
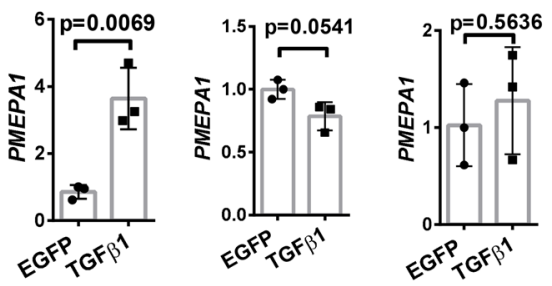

Fig. 7 PMEPA1 expression correlates with TGF $\beta$ expression and poor survival outcome in human HCC patients. a-c Spearman's correlation of PMEPA1 mRNA expression and $\mathbf{a} T G F \beta 1, \mathbf{b} T G F \beta 2$ and $\mathbf{c} T G F \beta 3$ mRNA levels in human HCCs showing a strong significant positive correlation. Images were obtained from the public cBioPortal website (https://www.cbioportal.org/). d High PMEPA1 expression $(N=274)$ showed a poor survival outcome when compared with low PMEPA1 expression $(N=91)$ in human HCC patients. Kaplan-Meier method and log-rank test were applied. $P=$ 0.033. e High PMEPA1 expression and c-MYC amplified patients (MYC ${ }^{A m p} ; N=45$ ) showed a poor survival outcome when compared with low PMEPA1 expression and MYC non-mutant (MYC ${ }^{W t} ; N=72$ ) HCC patients. Kaplan-Meier method and log-rank test were applied. $P=0.048$. $\mathbf{f}$ Western blot analysis showing the expression of c-MYC in human HCC cells. GAPDH was used as a loading control. $\mathbf{g}$ Western blot analysis showing the expression of p-SMAD2/3, SMAD2/3, and PMEPA1 in EGFP and TGF $\beta 1$ transfected human HCC cells. $\beta$-ACTIN was used as a loading control. $\mathbf{h}$ mRNA expression of PMEPA1 gene in EGFP or TGF $\beta 1$ transfected human HCC cells. Student $t$ test was applied for statistical analysis.

at the molecular level. Consistently, in human HCC samples, we discovered that TGF $\beta$ related downstream EMT targets such as $C R E B B P^{43}, E P 300^{44}, A P C^{45}, S K I L^{46}$, and MAP2K1 (MEK1) $)^{47}$ are not overexpressed in c-MYC amplified tumor samples based on TCGA LIHC data analysis (Supplementary Fig. 12). We found instead the 


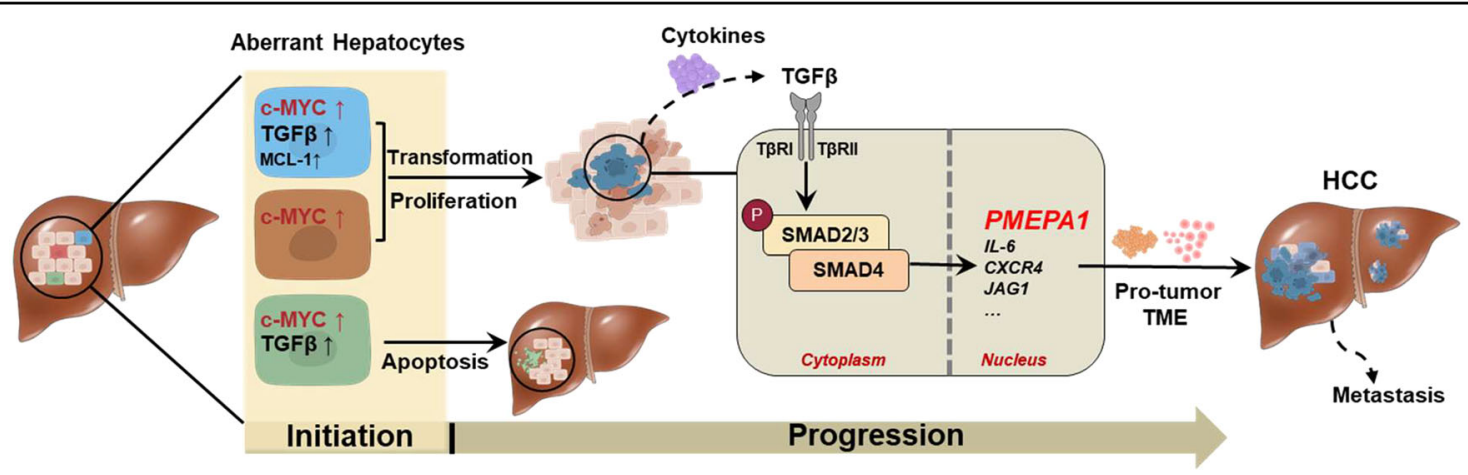

Fig. 8 Scheme showing the role of TGF $\beta$-SMAD signaling in the regulation c-Myc induced hepatocarcinogenesis. Abbreviation: TME, tumor microenvironment.

significant upregulation of inflammatory cytokines and angiogenesis related genes in vivo and in vitro when TGF $\beta 1$ was overexpressed in c-MYC HCC cells. Therefore, TGF $\beta$ regulated tumor microenvironment reprogramming might be the main mechanism whereby TGF $\beta$ promotes c-MYC HCC progression.

Our data also suggest that the TGF $\beta$ cascade may play a key role in modulating immune cells in HCC. Currently, immunotherapies, especially checkpoint inhibitors, have shown great promise ${ }^{48}$ as the first line treatment strategy against HCC. For instance, the IMBRAVE-150 clinical trial demonstrated the superior efficacy of anti-VEGF and anti-PDL1 antibodies for the treatment of advanced stage HCC than that of Sorafenib ${ }^{48}$. Nonetheless, the clinical trial also showed that most of the patients progressed under the combination immunotherapy ${ }^{48}$. Activated TGF $\beta$ has been proposed as a possible mediator of immunotherapy resistance ${ }^{49}$. Accordingly, a recent study from our group showed that c-Myc mouse HCCs were resistant to anti-PDL1 antibody treatment ${ }^{50}$. Thus, it would be of high importance to investigate whether the activated TGF $\beta$ signaling contributes to anti-PDL1 resistance in c-Myc mouse HCC. Furthermore, the combination of anti-PDL1 antibodies with TGF $\beta$ pathway inhibitors, such as Galunisertib ${ }^{51}$, should be tested and may be effective for HCC treatment. The c-Myc mouse model may represent an excellent preclinical tool to validate this hypothesis.

In this manuscript, we discovered PMEPA1 as a potential target of the TGF $\beta$ cascade in $\mathrm{c}-\mathrm{MYC}$ HCC. PMEPA1 is an androgen-regulated protein highly expressed in various solid tumors, including $\mathrm{HCC}^{52}$. PMEPA1 is known to play an important role downstream of the TGF $\beta$ signaling pathway in prostate ${ }^{53}$ and breast cancers $^{54}$. In a comprehensive genome-wide mouse HCC microarrays study, PEMPA1 was identified as a classifier for HCC with a late TGF $\beta$ signature, which accurately predicted liver metastasis ${ }^{55}$. However, the function of
PMEPA1 in HCC has not been established to date. Here, we show that high PMEPA1 expression correlates with poor survival outcome in human HCCs and targeting Pmepa1 inhibits TGF $\beta 1$ activated c-Myc murine HCC cells migration. Moreover, we found that PMEPA1 mRNA expression is upregulated in human HCC samples (Supplementary Fig. 13), and its level correlates with TGF $\beta$, TGF 32 , and TGF 33 mRNA expression in TCGA liver cancer samples (Fig. 7a). These findings are consistent with the data from Cao et al. ${ }^{36}$ using an independent HCC cohort. As TGF $\beta$ likely regulates tumor progression via multiple genes/pathways, and PMEPA1 may only represent one of these genes, further studies are needed, especially in vivo, to delineate the functional contribution of PMEPA1 in HCC progression. In the current study, we found that TGF $\beta 1$ activation induced upregulation of PMEPA1 in c-MYC high expressed human HCC cell lines, but not in c-MYC low expressed human HCC cell lines. The results support the hypothesis that PMEPA1 may be a major TGF $\beta$ effector and serve as a target for cMYC activated liver cancer treatment.

\section{Acknowledgements}

We sincerely thank Dr. Simon Weonsang Ro (Kyung Hee University, Seoul, Republic of Korea) for providing us with the pT3-GFP-shSmad2 and pT3-GFPshSmad3 plasmids.

\section{Author details}

'Liver Transplantation Division, Department of Liver Surgery, West China Hospital, Sichuan University, Chengdu, People's Republic of China.

${ }^{2}$ Department of Bioengineering and Therapeutic Sciences and Liver Center, University of California, San Francisco, CA, USA. ${ }^{3}$ Laboratory of Liver Surgery, West China Hospital, Sichuan University, Chengdu, Sichuan, People's Republic of China. ${ }^{4}$ Department of General Surgery, The Second Affiliated Hospital of Xi'an Jiaotong University, Xi'an Jiaotong University, Xi'an, People's Republic of China. Institute of Pathology, University of Regensburg, Regensburg, Germany

\section{Author contributions}

H.W., P.W., M.X., X.S. and H.W. provided acquisition, analysis and interpretation of data, and statistical analysis; Y.Z. and X.C. performed study concept and design; H.W., Y.Z., D.F.C. and X.C. performed development of methodology and writing, review and revision of the paper; M.E. provided technical and material support. All authors read and approved the final paper. 


\section{Ethics declarations}

Animal experiments were performed in accordance with protocols approved by UCSF Institutional Animal Care Use Committee (IACUC).

\section{Funding statement}

This study was supported by NIH grants R01CA190606 and R01CA239251 to XC, P30DK026743 for UCSF Liver Center, National Natural Science Foundation of China (grant number 82002967) to HW.

\section{Conflict of interest}

The authors declare no competing interests.

\section{Publisher's note}

Springer Nature remains neutral with regard to jurisdictional claims in published maps and institutional affiliations.

Supplementary information The online version contains supplementary material available at https://doi.org/10.1038/s41419-021-03488-z.

Received: 28 October 2020 Revised: 25 January 2021 Accepted: 27 January 2021

Published online: 19 February 2021

\section{References}

1. Ferlay, J. et al. Cancer incidence and mortality worldwide: Sources, methods and major patterns in GLOBOCAN 2012. Int. J. Cancer 136, E359-E386 (2015).

2. Couri, T. \& Pillai, A. Goals and targets for personalized therapy for HCC. Hepatol. Int. 13, 125-137 (2019).

3. El-Serag, H. B. \& Rudolph, K. L. Hepatocellular carcinoma: epidemiology and molecular carcinogenesis. Gastroenterology 132, 2557-2576 (2007).

4. Hernandez-Gea, V., Toffanin, S., Friedman, S. L. \& Llovet, J. M. Role of the microenvironment in the pathogenesis and treatment of hepatocellular carcinoma. Gastroenterology 144, 512-527 (2013).

5. Severi, T., van Malenstein, H., Verslype, C. \& van Pelt, J. F. Tumor initiation and progression in hepatocellular carcinoma: risk factors, classification, and therapeutic targets. Acta Pharmacol. Sin. 31, 1409-1420 (2010).

6. Chen, J. et al. Analysis of genomes and transcriptomes of hepatocellular carcinomas identifies mutations and gene expression changes in the transforming growth factor- $\beta$ pathway. Gastroenterology 154, 195-210 (2018).

7. Vander Ark, A., Cao, J. \& Li, X. TGF- $\beta$ receptors: In and beyond TGF- $\beta$ signaling Cell. Signal. 52, 112-120 (2018).

8. Hata A. \& Chen Y.-G. TGF- $\beta$ signaling from receptors to Smads. Cold Spring Harb. Perspect. Biol. 8, 1-31 (2016).

9. Derynck, R., Zhang, Y. \& Feng, X.-H. Transcriptional activators of TGF- $\beta$ responses: Smads. Cell 95, 737-740 (1998)

10. Morikawa M., Derynck R. \& Miyazono,K. TGF- $\beta$ and the TGF- $\beta$ family: contextdependent roles in cell and tissue physiology. Cold Spring Harb. Perspect. Biol. 8, 1-24 (2016).

11. Mancarella S. et al. Validation of Hepatocellular carcinoma experimental models for TGF- $\beta$ promoting tumor progression. Cancers 11, 1510-1529 (2019).

12. Tsubakihara Y. \& Moustakas A. Epithelial-mesenchymal transition and metastasis under the control of transforming growth factor $\beta$. Int. J. Mol. Sci. 19 3672-3701 (2018)

13. Ally, A. et al. Comprehensive and integrative genomic characterization of hepatocellular carcinoma. Cell 169, 1327-1341.e1323 (2017).

14. Liu, P. et al. A functional mammalian target of rapamycin complex 1 signaling is indispensable for c-Myc-driven hepatocarcinogenesis. Hepatology $\mathbf{6 6}$, 167-181 (2017)

15. Xin, B. et al. Critical role of Myc activation in mouse hepatocarcinogenesis induced by the activation of AKT and RAS pathways. Oncogene 36, 5087-5097 (2017).

16. Feng, X.-H., Liang, Y.-Y., Liang, M., Zhai, W. \& Lin, X. Direct interaction of C-Myc with Smad2 and Smad3 to inhibit TGF- $\beta$-mediated induction of the CDK inhibitor p15Ink4B. Mol. Cell 9, 133-143 (2002).
17. Moon, $\mathrm{H}$. et al. Transforming growth factor- $\beta$ promotes liver tumorigenesis in mice via up-regulation of Snail. Gastroenterology 153, 1378-1391 (2017).

18. $\mathrm{Hu}, \mathrm{J}$. et al. Co-activation of AKT and c-Met triggers rapid hepatocellular carcinoma development via the mTORC1/FASN pathway in mice. Sci. Rep. $\mathbf{6}$ 20484 (2016).

19. Chen, X. \& Calvisi, D. F. Hydrodynamic transfection for generation of novel mouse models for liver cancer research. Am. J. Pathol. 184, 912-923 (2014).

20. Soares K. C. et al. A preclinical murine model of hepatic metastases. JoVE $\mathbf{9 1}$ e51677 (2014).

21. Conesa, A. et al. A survey of best practices for RNA-seq data analysis. Genome Biol. 17, 13 (2016).

22. Hothorn, T. \& Zeileis, A. Generalized maximally selected statistics. Biometrics $\mathbf{6 4}$, 1263-1269 (2008).

23. Huang, Y. L. et al. Promising diagnostic and prognostic value of E2Fs in human hepatocellular carcinoma. Cancer Res. 78, 2305 (2018).

24. Zhang, Y.-L. et al. SPON2 promotes M1-like macrophage recruitment and inhibits hepatocellular carcinoma metastasis by distinct integrin-Rho GTPase-Hippo pathways. Cancer Res. 78, 2305 (2018).

25. Yang, D., Zhao, Y., Liu, J., Sun, Y. \& Jia, L. Protective autophagy induced by RBX1/ROC1 knockdown or CRL inactivation via modulating the DEPTORMTOR axis. Autophagy 8, 1856-1858 (2012).

26. Chen, H.F. et al. Role of a novel functional variant in the PPP2R1A Promoter on the regulation of PP2A-aalpha and the risk of hepatocellular carcinoma. PLOS ONE 8, e59574 (2013).

27. Tzavlaki K. \& Moustakas A. TGF- $\beta$ signaling. Biomolecules 10, 487-524 (2020).

28. Cao, Z. et al. MYC Phosphorylation, activation, and tumorigenic potential in hepatocellular carcinoma are regulated by HMG-CoA reductase. Cancer Res. 71, 2286 (2011)

29. Spicer, J. D. et al. Neutrophils promote liver metastasis via Mac-1-mediated interactions with circulating tumor cells. Cancer Res. 72, 3919 (2012).

30. $\mathrm{Ye}, \mathrm{X}$. et al. Distinct EMT programs control normal mammary stem cells and tumour-initiating cells. Nature 525, 256-260 (2015)

31. Wu, Z-Q. et al. Canonical Wnt suppressor, Axin2, promotes colon carcinoma oncogenic activity. Proc. Natl Acad. Sci. USA 109, 11312 (2012).

32. Hamidi, A. et al. TGF- $\beta$ promotes PI3K-AKT signaling and prostate cancer cell migration through the TRAF6-mediated ubiquitylation of p85a. Sci. Signal. 10, eaal4186 (2017).

33. $\mathrm{Ji}, \mathrm{H}$. et al. Proteome profiling of exosomes derived from human primary and metastatic colorectal cancer cells reveal differential expression of key metastatic factors and signal transduction components. Proteomics 13, 1672-1686 (2013).

34. Yang, L. TGF $\beta$ and cancer metastasis: an inflammation link. Cancer Metastasis Rev. 29, 263-271 (2010)

35. Caja L. et al. TGF- $\beta$ and the tissue microenvironment: relevance in fibrosis and cancer. Int. J. Mol. Sci. 19, 1294-1317 (2018).

36. Cao, Y. et al. NGS-based transcriptome profiling reveals biomarkers for companion diagnostics of the TGF- $\beta$ receptor blocker galunisertib in HCC. Cell Death Dis. 8, e2634-e2634 (2017).

37. Liu, X. et al. Focal adhesion kinase activation limits efficacy of Dasatinib in CMyc driven hepatocellular carcinoma. Cancer Med. 7, 6170-6181 (2018).

38. Lahsnig, C. et al. ILEl requires oncogenic Ras for the epithelial to mesenchymal transition of hepatocytes and liver carcinoma progression. Oncogene $\mathbf{2 8}$, 638-650 (2009).

39. $\mathrm{Mu}$, X. et al. TGF- $\beta$ signaling is often attenuated during hepatotumorigenesis, but is retained for the malignancy of hepatocellular carcinoma cells. PLOS ONE 8, e63436 (2013).

40. Dituri, F., Mancarella, S., Cigliano, A., Chieti, A. \& Giannelli, G. TGF- $\beta$ as multifaceted orchestrator in hcc progression: signaling, emt, immune microenvironment, and novel therapeutic perspectives. Semin Liver Dis. 1, 53-69 (2019).

41. Smith J. L. et al. YAP1 withdrawal in hepatoblastoma drives therapeutic differentiation of tumor cells to functional hepatocyte-like cells. Hepatology, https://doi.org/10.1002/hep.31389 (2020).

42. Moustakas A. \& Heldin C.-H. Mechanisms of TGFß-induced epithelialmesenchymal transition. J. Clin. Med. 5, 63-96 (2016).

43. Zhou, B. et al. Interactions between $\beta$-catenin and transforming growth factor$\beta$ signaling pathways mediate epithelial-mesenchymal transition and are dependent on the transcriptional co-activator CAMP-response elementbinding protein (CREB)-binding protein (CBP). J. Biol. Chem. 287, 7026-7038 (2012). 
44. Yokomizo, C. et al. High expression of p300 in HCC predicts shortened overall survival in association with enhanced epithelial mesenchymal transition of HCC cells. Cancer Lett. 310, 140-147 (2011).

45. Chaw, S. Y. et al. Epithelial to mesenchymal transition biomarkers E-cadherin, beta-catenin, APC and Vimentin in oral squamous cell carcinogenesis and transformation. Oral. Oncol. 48, 997-1006 (2012)

46. Sengupta, S., Jana, S., Biswas, S., Mandal, P. K. \& Bhattacharyya, A. Cooperative involvement of NFAT and SnoN mediates transforming growth factor- $\beta$ (TGF$\beta$ ) induced EMT in metastatic breast cancer (MDA-MB 231) cells. Clin. Exp. Metastasis 30, 1019-1031 (2013).

47. Ma, J. et al. BMP4 promotes oxaliplatin resistance by an induction of epithelialmesenchymal transition via MEK1/ERK/ELK1 signaling in hepatocellular carcinoma. Cancer Lett. 411, 117-129 (2017).

48. Finn, R. S. et al. Atezolizumab plus bevacizumab in unresectable hepatocellular carcinoma. N. Engl. J. Med. 382, 1894-1905 (2020).

49. Chen, J., Gingold, J. A. \& Su, X. Immunomodulatory TGF- $\beta$ signaling in hepatocellular carcinoma. Trends Mol. Med. 25, 1010-1023 (2019).
50. Shang R. et al. Cabozantinib-based combination therapy for the treatment of hepatocellular carcinoma. Gut, https://doi.org/10.1136/gutjnl-2020-320716 (2020).

51. Faivre, S. et al. Novel transforming growth factor beta receptor I kinase inhibitor galunisertib (LY2157299) in advanced hepatocellular carcinoma. Liver Int. 39, 1468-1477 (2019)

52. Brunschwig, E. B. et al. PMEPA1, a Transforming growth factor- $\beta$-induced marker of terminal colonocyte differentiation whose expression is maintained in primary and metastatic colon cancer. Cancer Res. 63, 1568 (2003).

53. Fournier, P. G. et al. The TGF- $\beta$ signaling regulator PMEPA1 suppresses prostate cancer metastases to bone. Cancer Cell 6, 809-821 (2015).

54. Zou, Y. et al. Transmembrane prostate androgen-induced protein 1 (PMEPA1) promotes the migration and maintains mesenchymal-like phenotype of breast cancer cells. Xi Bao Yu Fen. Zi Mian Yi Xue Za Zhi. 32, 158-162 (2016).

55. Coulouarn, C., Factor, V. M. \& Thorgeirsson, S. S. Transforming growth factor- $\beta$ gene expression signature in mouse hepatocytes predicts clinical outcome in human cancer. Hepatology 47, 2059-2067 (2008). 